\title{
Radiation and ATM inhibition: the heart of the matter
}

\author{
Ester M. Hammond and Ruth J. Muschel
}

Cancer Research UK/MRC Oxford Institute for Radiation Oncology, Department of Oncology, University of Oxford, Oxford, United Kingdom.

\begin{abstract}
Numerous in vitro studies have shown that human cell lines lacking functional ATM are extremely radiosensitive. In this issue, Moding et al. demonstrate using a murine model of sarcoma that deletion of the Atm gene has much less of a radiosensitizing effect on normal cardiac endothelia than on rapidly proliferating tumor endothelia. This work confounds our assumptions about the generality of the role of ATM in radiation sensitivity and the potential use of ATM inhibitors as radiosensitizers.
\end{abstract}

\section{The role of ATM in damage response}

The ataxia telangiectasia mutated (ATM) kinase is a member of the PI3K-like protein kinase (PIKK) family with extensive roles in DNA damage response signaling (reviewed in ref. 1). In addition, ATM can respond to non-DNA-damaging stresses and has recently been described as having a role in neoangiogenesis in a melanoma model (2-5). DNA damage leads to activation of ATM kinase activity and thus phosphorylation of a myriad of downstream targets, including p53, CHK2, and $\operatorname{KAP}-1(6,7)$. This activation triggers cell cycle checkpoints, arrest, and delays in the $G_{1}, S$, and $\mathrm{G}_{2}$ phases of the cell cycle and enables DNA repair of double-stranded breaks both by homologous recombination and by nonhomologous end joining $(8,9)$. Hence, fibroblasts and tumor cells are radiosensitized to X-ray radiation therapy (XRT) in culture by pharmacological ATM inhibition, or by ATM mutation and deletion (10). These data suggest that inhibition of ATM should radiosensitize tumors; however, there is a hesitancy to employ this strategy clinically, due to fears that normal tissues could be substantially sensitized as well. This reluctance is well founded, as it is based on the catastrophic response of patients with ataxia telangiectasia (AT) to XRT. The AT syndrome, which is now characterized in part by extreme radiosensitivity, is caused by mutations in the ATM gene. Before AT was fully characterized, a few patients who developed lymphoma (a frequent event in AT) were radiated to treat mediastinal cancers and suffered drastic side effects. Those few reports stressed severe mucositis of the esophagus and skin desquamation (e.g., ref. 11). This led to the subsequently proven supposition that ATM plays an important role in the response and repair of DNA damage, but also the understandable reticence of clinicians to consider XRT for AT patients and reluctance to use AT inhibitors because of the potential normal tissue sensitivity. While the clinical observations point to mucous membranes and skin as targets for deleterious normal tissue effects, it should be remembered that thoracic radiation also inevitably results in some radiation dose to the heart. Work over recent years has shown that irradiation of the heart during treatment for breast cancer leads to long-term increased risk for atherosclerotic coronary disease (12). However, this dose-dependent pathology evolves over the long term and is not replicated in mice unless they are also predisposed to atherosclerosis, as in mice with ApoE deficiency (13), and so might not emerge in some model systems.

\section{An elegant model to understand the role of ATM}

In this issue, Moding et al. used an innovative and intricate system to distinguish

Related Article: p. 3325

Conflict of interest: The authors have declared that no conflict of interest exists.

Reference information: / Clin Invest. 2014;124(8):3289-3291. doi:10.1172/JCI77195.

the effects of ATM deletion in endothelium from the heart and from tumors (14). The authors used a sarcoma model that they previously established in mice with mutant $\operatorname{Kras}^{G 12 D}$ and $p 53$ flanked by inactivating stop sequences that contain FlpO cleavage sites (Figure 1). Injection of FlpO-expressing adenovirus triggered deletion of the stop sequences, which led to expression of the $\mathrm{Kras}^{G 12 D}$ oncogene and deletion of the $p 53$ suppressor gene, resulting in sarcoma formation at the site of the injection. Conditional deletion of ATM from the vasculature was accomplished using an alternative recombinase system, the more commonly used Cre, induced in the vasculature by the introduction of a Cre transgene driven by the endothelial specific $V E$-Cadherin promoter. Thus, the vasculature of both the tumor and the normal heart lacked ATM in this sarcoma-bearing mouse model. Irradiation of the mouse chest delivered radiation to both the sarcoma and the heart, allowing for direct comparison of the radiation response of endothelial cells of the tumor and cardiac compartments.

Irradiation of the tumor, using a clinically relevant dose of $20 \mathrm{~Gy}$, led to increased levels of apoptosis in the ATMdeleted tumor endothelial compartment and decreased perfusion in the irradiated tumor. Together, these data indicate that ATM affected the radiation response of the tumor endothelial cells. It should be noted that a distinction between functional impairment strictly due to vascular destruction and the capacity for vascular regrowth is not separable in these experiments. These tumors (lacking ATM in the endothelium) had a significant growth delay after irradiation, but not before, therefore demonstrating that targeting tumor endothelial cells is an effective means of increasing radiation-induced loss of viability. In noteworthy contrast, the irradiated heart showed no decrement in function or increase in necrosis regardless of endothelial ATM status. This finding contrasted a previous report from this group that radiation of the hearts of mice 
A
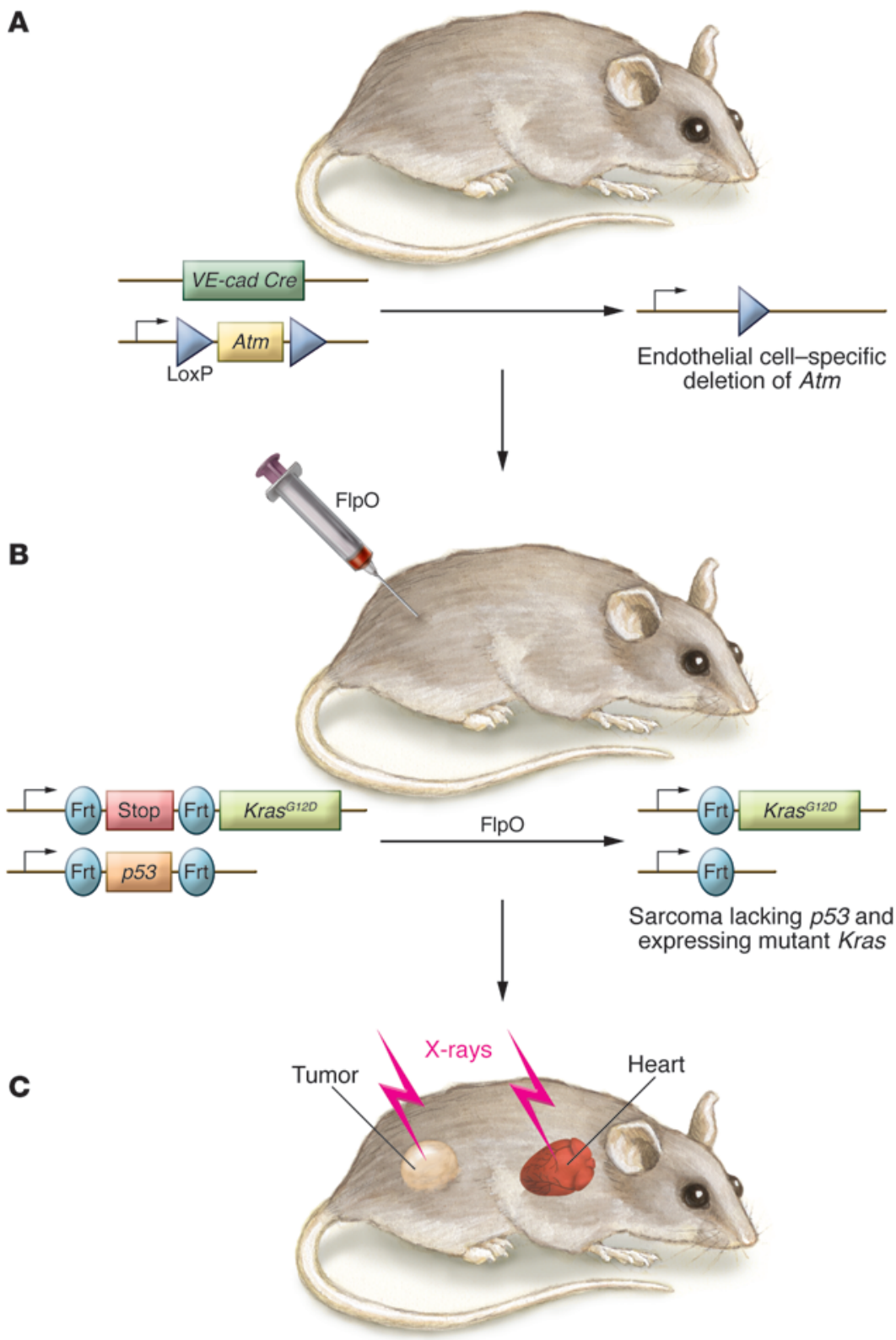

All endothelial cells (cardiac and tumor): $\mathrm{Kras}^{+/ / 4}, \mathrm{p53}^{+/ 4}, \mathrm{Atm}^{-/}$ Sarcoma: Kras ${ }^{G 12 D}, \mathrm{p}^{-/ /}, \mathrm{Atm}^{+/+}$
Figure 1. Generation of genetically engineered mice with sarcoma driven by deletion of $p 53$ and expression of mutant Kras in mice with endothelial cell-specific ATM loss. (A) Moding et al. developed this model (14) using genetically engineered mice in which Atm was selectively deleted in the vast majority of endothelial cells due to transgenic expression of the recombinase Cre from the endothelial-selective VE-cadherin promoter. (B) Subsequently, a sarcoma was induced in these mice. In addition to the alterations of the Atm gene, these mice had $p 53$ flanked by Frt sequences that can be cleaved and deleted by a different recombinase, FlpO. The mice further contained a mutant Kras that is inhibited in its expression by stop sequences that also can be cleaved and deleted by FlpO. Injection of an adenovirus expressing FlpO resulted in expression of oncogenic Kras and loss of p53 and led to sarcoma formation at the site of the injection. Notably, the tumor itself expressed wild-type ATM. (C) Finally, both the tumor and the heart were irradiated, allowing for comparison of tumor endothelial and cardiac endothelial response to radiation. with VE-Cadherin-Cre-mediated p53 deletion or of mice lacking $p 21$ sensitized them to myocardial necrosis within four months after 12 Gy whole-heart irradiation (15).

\section{Role of proliferation in sensitivity to Atm loss}

As a result of these data, Moding et al. hypothesized that the difference between the tumor and cardiac endothelium might be that the endothelium in the tumor is significantly more proliferative than the mainly quiescent adult cardiac endothelium (14). In vivo, they addressed this question by administering SCH72765, an inhibitor of cyclin-dependent kinase 1 (CDK1), CDK2, CDK5, and CDK9 that inhibits cell cycle progression. After treatment of the sarcoma-bearing mice with SCH72765, the level of cell death after irradiation was greatly diminished. Further testing of this hypothesis in tissue culture was confounded by the fact that the ATM-deleted endothelial cells did not grow well enough in culture to perform these experiments unless p53 was also deficient. Perhaps the combination of functional p53 and the absence of ATM allows activation of checkpoints and amelioration of radiation damage. Hence, the authors compared endothelial cells in culture with p53 deficiency. Recognizing that there are caveats in examining ATMdeficient cells in culture that also lack p53, Moding et al. reported that ATM deficiency resulted in endothelial cells that were more sensitive to radiation in clonogenic survival assays (14). After inhibition of proliferation via SCH72765 treatment and irradiation, ATM status did not alter cell death or micronucleus formation, which suggests that ATM in endothelial cells was immaterial if a cell cycle block was present at the time of irradiation. These data led to the conclusion that loss 
of Atm in endothelial cells only increases radiosensitivity if the cells are transiting through the cell cycle.

These data are consistent with the importance of ATM signaling for cell cycle arrest, including $G_{1}, S$, and $G_{2}$ checkpoints, and the concept that these delays might allow sufficient time for DNA repair. The pharmacological CDK inhibitor could be postulated to substitute for the presence of active ATM in the ATM-deficient endothelium. However, ATM has also been shown to signal in the mechanistic pathways for both homologous recombination and nonhomologous end-joining pathways for DNA repair. In fact, inhibition of proliferation of ATM-deficient fibroblasts in tissue culture by contact inhibition did not reduce their radiosensitization substantially compared with ATM-proficient cells (16). Additional experiments will be required to determine whether proliferation is the only factor determining altered radiosensitivity in endothelial cells.

\section{Conclusions and future directions}

Radiation therapy is frequently used in cancer treatment, leading to the objective of many researchers to devise means to improve the sensitivity of the tumor $(17,18)$. DNA double-strand breaks are regarded as the predominant lethal lesion induced by irradiation; thus, for many years it has been hypothesized that inhibition of DNA damage response signaling/DNA repair could lead to more effective radiation treatment. The current paper (14) adds considerable information to the preclinical work regarding such strategies. This study will encourage further investigation of the use of pharmacological inhibition of ATM (as opposed to genetic knockout) as a means of radiosensitization clinically. Given that ATM is a large protein with extensive regions of unknown function, it is possible that inhibition of its kinase activity may not entirely recapitulate the absence of the protein. Equally possible are confounding effects as a result of either incomplete ATM inhibition or off-target effects, for example, activity against other PIKK family members. It is also worth not- ing that in recent years, certainly since the tragic irradiation of AT patients, radiation technologies have improved markedly, so that radiation doses can now be delivered with greater precision, increasingly reducing the dose to normal tissue. For example, the possibility of using ATM inhibitors in combination with radiotherapy for treatment of glioblastoma - which arise in the brain, a region of low proliferation - has already begun to be explored $(19,20)$. An ATM inhibitor was also found to sensitize tumor xenografts to the DNA-damaging cancer therapeutic drugs etoposide and irinotecan, without any reported normal tissue toxicity (21).

In conclusion, ATM is well known to be one of the master regulators of the cellular response to radiation-induced DNA damage and a key determinant of radiosensitivity. This set of experiments demonstrated that different tissues may have different responses to ATM inhibition. Whether this is simply due to differences in proliferation or whether additional factors are relevant remains to be determined. Understanding of these factors could point the way to the clinical use of ATM inhibitors as radiosensitizers in cancer therapy.

\section{Acknowledgments}

E.M. Hammond and R.J. Muschel are supported by CRUK, MRC, and EPSRC.

Address correspondence to: Ruth J. Muschel, Department of Oncology, University of Oxford, ORCRB, Roosevelt Drive, Oxford OX37DQ, United Kingdom. Phone: 44.1865225847; E-mail: ruth.muschel@ oncology.ox.ac.uk.

1. Shiloh Y, Ziv Y. The ATM protein kinase: regulating the cellular response to genotoxic stress, and more. Nat Rev Mol Cell Biol. 2013;14(4):197-210.

2. Okuno Y, Nakamura-Ishizu A, Otsu K, Suda T, Kubota Y. Pathological neoangiogenesis depends on oxidative stress regulation by ATM. Nat Med. 2012;18(8):1208-1216.

3. Olcina MM, et al. Replication stress and chromatin context link ATM activation to a role in DNA replication. Mol Cell. 2013;52(5):758-766.

4. Bakkenist CJ, Kastan MB. DNA damage activates ATM through intermolecular autophosphorylation and dimer dissociation. Nature. 2003;421(6922):499-506.
5. Guo Z, Deshpande R, Paull TT. ATM activation in the presence of oxidative stress. Cell Cycle. 2010;9(24):4805-4811.

6. Goodarzi AA, et al. ATM signaling facilitates repair of DNA double-strand breaks associated with heterochromatin. Mol Cell. 2008;31(2):167-177.

7. Matsuoka S, et al. ATM and ATR substrate analysis reveals extensive protein networks responsive to DNA damage. Science. 2007;316(5828):1160-1166.

8. Helleday T, Petermann E, Lundin C, Hodgson $\mathrm{B}$, Sharma RA. DNA repair pathways as targets for cancer therapy. Nat Rev Cancer. 2008;8(3):193-204.

9. Jackson SP, Bartek J. The DNA-damage response in human biology and disease. Nature. 2009;461(7267):1071-1078.

10. Hickson I, et al. Identification and characterization of a novel and specific inhibitor of the ataxia-telangiectasia mutated kinase ATM. Cancer Res. 2004;64(24):9152-9159.

11. Gotoff SP, Amirmokri E, Liebner EJ. Ataxia telangiectasia. Neoplasia, untoward response to $\mathrm{x}$-irradiation, and tuberous sclerosis. Am J Dis Child. 1967;114(6):617-625.

12. Darby SC, et al. Risk of ischemic heart disease in women after radiotherapy for breast cancer. NEngl J Med. 2013;368(11):987-998.

13. Gabriels K, et al. Local heart irradiation of ApoE(-/-) mice induces microvascular and endocardial damage and accelerates coronary atherosclerosis. Radiother Oncol. 2012;105(3):358-364.

14. Moding EJ, et al. Atm deletion with dual recombinase technology preferentially radiosensitizes tumor endothelium. J Clin Invest. 2014;124(8):3325-3338.

15. Lee CL, et al. p53 functions in endothelial cells to prevent radiation-induced myocardial injury in mice. Sci Signal. 2012;5(234):ra52.

16. Kuhne M, Riballo E, Rief N, Rothkamm K, Jeggo PA, Lobrich M. A double-strand break repair defect in ATM-deficient cells contributes to radiosensitivity. Cancer Res. 2004;64(2):500-508.

17. Begg AC, Stewart FA, Vens C. Strategies to improve radiotherapy with targeted drugs. Nat Rev Cancer. 2011;11(4):239-253.

18. Delaney G, Jacob S, Featherstone C, Barton M. The role of radiotherapy in cancer treatment: estimating optimal utilization from a review of evidence-based clinical guidelines. Cancer. 2005;104(6):1129-1137.

19. Biddlestone-Thorpe L, et al. ATM kinase inhibition preferentially sensitizes p53-mutant glioma to ionizing radiation. Clin Cancer Res. 2013;19(12):3189-3200.

20. Golding SE, et al. Dynamic inhibition of ATM kinase provides a strategy for glioblastoma multiforme radiosensitization and growth control. Cell Cycle. 2012;11(6):1167-1173.

21. Batey MA, et al. Preclinical evaluation of a nove ATM inhibitor, KU59403, in vitro and in vivo in p53 functional and dysfunctional models of human cancer. Mol Cancer Ther. 2013;12(6):959-967. 